\title{
THE CORRELATION FUNCTION OF CLUSTERS OF GALAXIES AND THE AMPLITUDE OF MASS FLUCTUATIONS IN THE UNIVERSE
}

\author{
H.J. Mo, Y.P. Jing, S.D.M. White \\ Max-Planck-Institut für Astrophysik \\ Karl-Schwarzschild-Strasse 1 \\ 85748 Garching, Germany
}

MNRAS: submitted 


\begin{abstract}
We show that if a sample of galaxy clusters is complete above some mass threshold, then hierarchical clustering theories for structure formation predict its autocorrelation function to be determined purely by the cluster abundance and by the spectrum of linear density fluctuations. Thus if the shape of the initial fluctuation spectrum is known, its amplitude $\sigma_{8}$ can be estimated directly from the correlation length of a cluster sample in a way which is independent of the value of $\Omega_{0}$. If the cluster mass corresponding to the sample threshold is also known, it provides an independent estimate of the quantity $\sigma_{8} \Omega_{0}^{0.6}$. Thus cluster data should allow both $\sigma_{8}$ and $\Omega_{0}$ to be determined observationally. We explore these questions using N-body simulations together with a simple but accurate analytical model based on extensions of Press-Schechter theory. Applying our results to currently available data we find that if the linear fluctuation spectrum has a shape similar to that suggested by the APM galaxy survey, then a correlation length $r_{0}$ in excess of $20 h^{-1} \mathrm{Mpc}$ for Abell clusters would require $\sigma_{8}>1$, while $r_{0}<15 h^{-1} \mathrm{Mpc}$ would require $\sigma_{8}<0.5$. With conventional estimates of the relevant mass threshold these imply $\Omega_{0} \lesssim 0.3$ and $\Omega_{0} \gtrsim 1$ respectively.
\end{abstract}

Key words: galaxies: clustering-cosmology: theory-dark matter

\title{
1. INTRODUCTION
}

Observations have shown that clusters of galaxies are strongly clustered in space. The cluster-cluster two-point correlation function, $\xi_{\mathrm{cc}}(r)$, is roughly a power law, $\xi_{\text {cc }}(r)=\left(r_{0} / r\right)^{\alpha}$, with $\alpha \sim 1.8$ and with a correlation length $r_{0}$ much larger than that of galaxies (see Bahcall 1988 for a review). The exact value of $r_{0}$ is, however, still controversial. Redshift surveys of clusters from 
the Abell catalogue (Abell 1958) and its southern extension (Abell, Corwin \& Olowin 1989, ACO) give $r_{0} \sim 20-25 h^{-1} \mathrm{Mpc}$ (Bahcall \& Soneira 1983; Klypin \& Kopylov 1983; Postman, Huchra \& Geller 1992; Peacock \& West 1992). These data also show that the correlation length $r_{0}$ increases with cluster richness $\mathcal{R}$ according to a scaling relation $r_{0} \approx 0.4 d$, where $d$ is the mean intercluster separation and is related to the mean space density $n$ by $d=n^{-1 / 3}$ (Bahcall \& Burgett 1986; Bahcall \& West 1992). The reliability of these results has, however, been questioned by a number of authors (e.g. Sutherland 1988; Dekel et al. 1989; Efstathiou et al. 1992b) who argued that they may be affected by projection effects intrinsic to the construction of the Abell/ACO catalogues. These authors claim that the correlation function of $\mathcal{R} \geq 1$ Abell clusters may have been significantly overestimated. Other authors (e.g. Bahcall \& West 1992; Jing, Plionis \& Valdarnini 1992; Peacock \& West 1992) claim that any bias in $\xi_{\text {cc }}$ due to projection effects is small. Recent estimates of $r_{0}$ based on the APM cluster catalogue (Dalton et al. 1992, 1994) and the Edinburgh-Durham cluster catalogue (Nichol et al. 1992) give $r_{0}=13-16 h^{-1} \mathrm{Mpc}$ for clusters with $d \approx 35-45 h^{-1} \mathrm{Mpc}$ (compared to $d \approx 55 h^{-1} \mathrm{Mpc}$ for $\mathcal{R} \geq 1$ Abell clusters). These results are still consistent with the $r_{0}-d$ relation described above. Since these catalogues are too small to give an accurate estimate of $r_{0}$ for richer clusters, the controversy over the correlation length of rich Abell clusters is still unresolved.

Despite the uncertainties in current observational results, the correlation function of clusters of galaxies remains one of the most important diagnostics for models of structure formation. Indeed, the strong observed correlation of clusters on large scales is very difficult to reconcile with the standard cold dark matter (CDM) model (White et al. 1987; Dalton et al. 1992; Jing et al.

\footnotetext{
${ }^{1}$ Throughout this paper, we write the Hubble constant as $H_{0}=100 h \mathrm{~km} \mathrm{~s}^{-1} \mathrm{Mpc}^{-1}$
} 
1993; Mo, Peacock \& Xia 1993; Dalton et al. 1994). In the near future, as new data from x-ray observations and large digital sky surveys become available, cluster correlation functions will provide much more stringent constraints on models. In addition, future observations of cluster x-ray properties and of gravitational lensing by clusters will provide improved estimates for the masses of clusters. We can then examine $\xi_{\mathrm{cc}}$ as an explicit function of cluster mass.

It is clearly important to understand how cosmological models are constrained by such observations. In this context, a simple analytic model for $\xi_{\text {cc }}$ and for cluster abundances is particularly desirable.

The observed high amplitude of $\xi_{\mathrm{cc}}$ is usually considered to be a result of clusters being high peaks in the initial density field (Kaiser 1984). Although qualitatively correct, this peak theory does not provide an adequate estimate for the two-point correlation functions of clusters in N-body simulations (Croft \& Efstathiou 1994; Mann, Heavens \& Peacock 1993). Furthermore, it is not clear how to derive a mass function for clusters from the peak theory, because peaks of small masses may be contained by those of larger masses.

An alternative scheme, the Press-Schechter formalism (Press \& Schechter 1974, PS) defines objects (halos) to be virialized structures, identified at the present time, which have grown from Gaussian initial density fluctuations. Although the original derivation of PS is flawed and recent rederivations are still far from rigorous (Bower 1991; Bond et al. 1991), the PS formalism gives surprisingly good fits to the mass functions of dark matter halos identified in N-body simulations of hierarchical clustering (Efstathiou et al. 1988; White, Efstathiou \& Frenk 1993; Lacey \& Cole 1994). Recently, Mo \& White (1995) show that the PS formalism and its extensions can also be used to derive a model for the spatial correlation of dark matter halos in hierarchical models. 
They find that the halo-halo two-point correlation functions predicted by this model agree surprisingly well with those derived from N-body simulations of evolution from scale-free initial power spectra. Such a model can obviously help us to understand better the clustering properties of clusters of galaxies. A related model has been used by Kashlinsky (1987) to explain the richness dependence of cluster correlations.

In this paper, we present additional tests of the model of Mo \& White for realistic initial power spectra and in the regime relevant to clusters of galaxies. We also discuss how these results can be used to relate observations of the large-scale clustering of clusters to the fundamental parameters of cosmology. We describe our model in Section 2. The N-body simulations used to test our model are described in Section 3. Comparisons of model predictions with simulation results are presented in Section 4. In Section 5 we apply our model to real clusters. A brief discussion of our results is given in Section 6.

\section{THE MODEL}

In this paper, we consider models in which the universe is spatially flat (so that $\Omega_{0}+\Omega_{\Lambda}=1$, where $\Omega_{0}$ and $\Omega_{\Lambda}$ are cosmic density parameters referring to matter and to the cosmological constant respectively) and dominated by cold dark matter. We also assume that the primordial density field is Gaussian, with a scale-invariant power spectrum. Neglecting the contribution of baryonic matter to $\Omega_{0}$, the initial power spectrum of density fluctuations can be written as (see Bardeen et al. 1986, equation G3):

$$
P(k) \propto k T^{2}(k),
$$


with

$$
T(k)=\frac{\ln (1+2.34 q)}{2.34 q}\left[1+3.89 q+(16.1 q)^{2}+(5.46 q)^{3}+(6.71 q)^{4}\right]^{-1 / 4}
$$

and

$$
q \equiv \frac{k}{\Gamma h \mathrm{Mpc}^{-1}},
$$

where, following Efstathiou et al. (1992a), we have introduced a shape parameter, $\Gamma \equiv \Omega_{0} h$, for the power spectrum.

A useful function equivalent to the power spectrum is the rms mass fluctuation in a spherical top-hat window of radius $R$ :

$$
\sigma^{2}(R)=\sum_{\mathbf{k}} P(k) \hat{W}^{2}(R ; k),
$$

where $\hat{W}(R, k)$ is the Fourier transform of the top-hat window. We normalize the power spectrum by specifying $\sigma_{8} \equiv \sigma\left(8 h^{-1} \mathrm{Mpc}\right)$. We define a mass parameter $M_{8}$ as the mean mass contained in a sphere with radius $8 h^{-1} \mathrm{Mpc}$ in a universe with the critical density, thus $M_{8}=5.8 \times 10^{14} h^{-1} M_{\odot}$. When lengths are written in units of $h^{-1} \mathrm{Mpc}$ and masses in units of $M_{8}$, all quantities we are interested in are independent of the Hubble constant. A model will then be specified by giving $\left(\Omega_{0}, \Gamma, \sigma_{8}\right)$.

In the PS formalism, dark matter halos are defined as spherically symmetric, virialized clumps of dark matter particles. The mass $M$ of a halo is related to its initial comoving radius $R$ (measured in current units) by

$$
M=\frac{4 \pi}{3} \bar{\rho} R^{3},
$$

where $\bar{\rho}$ is the mean mass density of the universe at present time. Thus the rms mass fluctuation on the scale of the halo is $\sigma(R)$. The fraction of matter in halos with mass exceeding $M$ is

$$
\mathcal{F}(\nu)=\operatorname{erfc}\left[\frac{\nu}{\sqrt{2}}\right],
$$


where $\nu \equiv \delta_{c} / \sigma(R)$ and $\operatorname{erfc}(x)$ is the complementary error function. The critical linear overdensity for virialization, $\delta_{c}$, is chosen to be $\delta_{c}=1.69$, irrespective of $\Omega_{0}$, as discussed in White et al. (1993, hereafter WEF). The PS formula for the comoving number of halos with mass $M$ is

$$
n(M) \mathrm{d} M=-\left(\frac{2}{\pi}\right)^{1 / 2} \frac{\bar{\rho}}{M} \frac{\delta_{c}}{\sigma} \frac{\mathrm{d} \ln \sigma}{\mathrm{d} \ln M} \exp \left[-\frac{\delta_{c}^{2}}{2 \sigma^{2}}\right] \frac{\mathrm{d} M}{M} .
$$

It follows that the total number density of halos with mass exceeding $M$ is

$$
n(>M)=-\frac{3}{(2 \pi)^{3 / 2}} \int_{R}^{\infty} \frac{1}{x^{3}} \frac{\delta_{c}}{\sigma(x)} \frac{\mathrm{d} \ln \sigma}{\mathrm{d} \ln x} \exp \left[-\frac{\delta_{c}^{2}}{2 \sigma^{2}}\right] \frac{\mathrm{d} x}{x} .
$$

Thus for given $\sigma(R)$ the abundance of halos depends only on $R$. An $\Omega_{0^{-}}$ dependence enters only through the relation between $R$ and $M$.

According to Mo \& White (1995), the two-point correlation function of dark matter halos with mass $M$ is related to that of the mass, $\xi_{\mathrm{m}}$, by

$$
\xi(r)=[b(M)]^{2} \xi_{\mathrm{m}}(r),
$$

where

$$
b(M)=1+\frac{1}{\delta_{c}}\left(\nu^{2}-1\right) .
$$

This is similar to what one gets from the peak-background split argument (Efstathiou et al. 1988; Cole \& Kaiser 1989). When halos with a range of masses are considered, $b(M)$ in equation $(7 \mathrm{~b})$ should be replaced by its average [weighted by $n(M)$ ] over $M$. Using N-body simulations of scale-free spectra, Mo \& White show that equation (7) holds even for $\xi_{\mathrm{m}}(r) \gtrsim 1$, as long as $r$ is not smaller than the Lagrangian radius $R$ of halos. For rich clusters of galaxies, this Lagrangian radius is $R \sim 10 h^{-1} \mathrm{Mpc}$ (WEF). Thus we expect equation (7) to be valid for $\xi_{\mathrm{cc}}(r)$ on $r \gtrsim 10 h^{-1} \mathrm{Mpc}$. On these scales, $\xi_{\mathrm{m}}(r)$ can be represented sufficiently accurately for our purposes by the 
Fourier transform of the linear power spectrum. For a given shape of power spectrum, the abundance of clusters gives a relation between the threshold $R$ and the amplitude $\sigma_{8}$ (through equation 6). The autocorrelation function gives another such relation through the definition $\nu \equiv \delta_{c} / \sigma(R)$ and equation (7). These two relations determine both $R$ and $\sigma_{8}$. An estimate of the cluster mass at threshold then determines $\Omega_{0}$

\section{N-BODY SIMULATIONS}

We use four sets of $\mathrm{P}^{3} \mathrm{M}$ N-body simulations to test the theories presented in the previous section. Each simulation can be characterized by three model parameters $\left(\Omega_{0}, \Gamma, \sigma_{8}\right)$ (as discussed in Section 2), and three simulation parameters: the box size $L$ (in $h^{-1} \mathrm{Mpc}$ ), the number of simulation particles $N$ and the effective force resolution $\eta$ (in $h^{-1} \mathrm{Mpc}$ ). For the first set of simulations, we use $\left(\Omega_{0}, \Gamma, \sigma_{8}\right)=(0.3,0.225,1)$ and $(L, N, \eta)=\left(400,100^{3}, 0.2\right)$. Six realizations were run for this set. The second set consists of five realizations of the SCDM universe with $\left(\Omega_{0}, \Gamma, \sigma_{8}\right)=(1.0,0.5,1.24)$ and $(L, N, \eta)=$ $\left(300,128^{3}, 0.23\right)$. The third is a single realization with $\left(\Omega_{0}, \Gamma\right)=(1,0.2)$ and $(L, N, \eta)=\left(256,128^{3}, 0.2\right)$. Analysis is made for two output times with $\sigma_{8}=1$ and $\sigma_{8}=0.5$. The final set is another single simulation with the same initial density spectrum (i.e. with $\Gamma=0.2$ ) and the same simulation parameters as the third set, but for a low density flat universe with $\Omega_{0}=0.2$. The two output times for this case correspond to $\sigma_{8}=1.07$ and 0.5 , respectively. The mass of each individual particle is $5.3 \times 10^{12} M_{\odot}, 3.6 \times 10^{12} M_{\odot}$, $2.2 \times 10^{12} M_{\odot}$ and $4.5 \times 10^{11} M_{\odot}$ in these four sets of simulations.

\section{COMPARISON WITH N-BODY SIMULATIONS}

For most of our discussion, we will use clusters identified by the standard 
friends-of-friends (FOF) group finder. A cluster in a simulation is then defined as all mass particles that are connected by joining particle pairs with separations smaller than a given linkage length, $l$, in units of the mean interparticle separation. There is no a priori reason for a particular choice of $l$. Following common practice, we choose $l=0.2$ (see e.g. Davis et al. 1985; Efstathiou et al. 1988; Lacey \& Cole 1994). The mean density within a cluster $(\bar{\delta})$ is then of the order 200, in rough agreement with what one obtains for a spherically symmetric, virialized object in an Einstein-de Sitter universe (Gunn \& Gott 1972). For a spatially flat universe with $\Omega_{0}=0.2, \bar{\delta}$ predicted by the virialization model is about 2.5 times higher (WEF), and a smaller linkage length might seem more reasonable. However, the real virialization process must be much more complicated than a spherical accretion model, and the appropriate value of $l$ may depend on cluster density profiles, which are different in different models (e.g. Jing et al. 1995). In the following we will see that our choice of $l$ gives a mass function that is in good agreement with that predicted by the PS formalism, even for a low-density flat universe. For comparison, we will also present some results obtained using the spherical-overdensity (SO) grouping algorithm invented by Lacey \& Cole. This algorithm is based on finding spherical regions with a certain predefined mean overdensity $\kappa$. A local density near each particle is needed to provide an initial list of possible halo centres, and is defined as $3(N+1) /\left(4 \pi r_{N}^{3}\right)$, where $r_{N}$ is the distance to the N'th nearest neighbour. Further details may be found in Lacey \& Cole (1994). We follow them in choosing $\kappa=180$ and $N=10$. In this algorithm the mass of a cluster is simply the number of particles within the bounding sphere. 


\subsection{Cluster number densities}

In Figure 1 the cumulative number densities of clusters in the simulations (circles) are compared directly with the predictions of the PS formalism (dashed curves). The error bars in Fig.1a and $1 \mathrm{~b}$ represent the $1 \sigma$ standard deviations between different realizations, whereas those in Fig.1c and 1d represent $1 \sigma$ Poisson fluctuations of the cumulative number density. This figure shows that the model prediction agrees with the simulation results reasonably well over a wide range of masses. The abundance range relevant for clusters of galaxies is $n(>M)=10^{-4.5}-10^{-6}\left(h^{-1} \mathrm{Mpc}\right)^{-3}$. The model works extremely well for these abundances. This is a nontrivial result, because the same $\delta_{c}$ and $l$ have been used for all cases. We have tried using $l=0.15$ for models with $\Omega_{0}=0.2$, and found that the agreement between the resulting mass function and model prediction is considerably worse. For comparison, we also show in Fig.1c and 1d the results for clusters identified by the SO group finder (crosses). It is clear that the mass function given by this group finder is similar to that given by the FOF group finder with linkage radius $l=0.2$. This result is in agreement with that obtained by Lacey \& Cole (1994) for scale-free initial density spectra evolved in an Einstein-de Sitter universe.

\subsection{The two-point correlation function}

The two-point correlation function of clusters in the simulations is estimated directly from pair counts:

$$
\xi_{\mathrm{cc}}(r)=\frac{\Delta P(r)}{4 \pi n r^{2} \Delta r}-1
$$

where $\Delta P(r)$ is the average number of neighbors, per cluster, with separation

in the range $r \pm \Delta r / 2$ and $n$ is the mean number density of clusters in the 
sample. The circles in Figures 2-7 show $\xi_{\text {cc }}$ for the FOF clusters identified in the N-body simulations. Results are shown for clusters in different mass bins, as indicated in the panels by the number of mass particles, $N_{p}$. The corresponding masses of clusters can be obtained by multiplying by the mass of each individual particle. The error bars in Figs. 2 and 3 represent the $1 \sigma$ scatter between realizations, while those in Figs.4-7 represents a $1 \sigma$ error based on Poisson statistics: $\Delta \xi_{\mathrm{cc}}(r)=[\Delta P(r)]^{1 / 2} /\left[4 \pi n r^{2} \Delta r\right]$. For comparison, we show as solid curves the fits of the data points at $r>10 h^{-1} \mathrm{Mpc}$ to a model, $\xi_{\mathrm{cc}}(r)=A \xi_{\mathrm{m}}(r)$, where $A$ is constant. In this fitting, each data point is weighted by the inverse of its bootstrap error, as is discussed in Mo, Jing \& Börner (1992). The crosses in Figs.4-7 show $\xi_{\text {cc }}$ for SO clusters with the same mass as the corresponding FOF clusters shown in the same panel. In agreement with the results obtained by Mo \& White (1995) for scale-free initial density spectra, the correlation functions for FOF and SO clusters of the same mass are quite similar. The dashed curves in Figs.2-7 show the predictions of equation (7). The figures show that theory and simulations agree remarkably well for all cases.

\section{APPLICATION TO REAL CLUSTERS}

As shown in Section 2, for a given shape of power spectrum the autocorrelation function and the number density of clusters with Lagrangian scale exceeding $R$ are both determined purely by $\sigma_{8}$. A comparison of the two allows $R$ to be eliminated, determining $\sigma_{8}$ in a way which is independent of $\Omega_{0}$.

Figure 8 shows the relation between the correlation length $r_{0}$ and the intercluster separation $d$ for two sets of linear power spectra. One has $\Gamma=0.5$ (Fig.8a), as in the standard CDM model, the other has $\Gamma=0.2$ (Fig.8b), 
which is similar to the shape obtained from the angular two-point correlation function of galaxies in the APM survey (see Efstathiou, Sutherland \& Maddox, 1990). It is clear that a stringent constraint on $\sigma_{8}$ can be obtained from an accurate measurement of $r_{0}$. The squares are observational results for different cluster samples as compiled by Bahcall \& Cen (1992), while the cross shows the result of a recent analysis by Dalton et al. (1994). The data point at $d=94 h^{-1} \mathrm{Mpc}$ is based on Abell $\mathcal{R} \geq 2$ clusters, and is very uncertain due to the small size of the sample (Peacock \& West 1992). As one can see from Fig.8a, the SCDM model with $\sigma_{8}=0.5$ does not have enough large-scale power to match the observational data. Indeed, if $r_{0} \geq 20 h^{-1} \mathrm{Mpc}$ for clusters with $d \sim 55 h^{-1} \mathrm{Mpc}$, then SCDM models with any reasonable $\sigma_{8}$ can be ruled out. In contrast, models with $\Gamma=0.2$ are consistent with such correlation lengths provided $\sigma_{8} \gtrsim 1$ (Fig.8b). As one can see from Fig.8b, in order to obtain an accurate value of $\sigma_{8}$, it is crucial to measure $r_{0}$ accurately for such rich clusters. If $r_{0}$ for clusters with $d \approx 55 h^{-1} \mathrm{Mpc}$ is actually significantly overestimated and its true value is smaller than $15 h^{-1} \mathrm{Mpc}$ (Sutherland 1988; Efstathiou et al. 1992b), then $\sigma_{8}<0.5$ is required for the power spectrum shape inferred from the APM galaxy sample. At present, the observational data are too uncertain to provide reliable constraints. In the future, as new data from digital sky surveys and x-ray observations become available, these arguments should provide a determination of $\sigma_{8}$ which can be compared directly with the amplitude on larger scales inferred from the COBE measurements of cosmic microwave background anisotropy (Smoot et al. 1992; Wright et al. 1994).

Based on the observed masses and abundances of rich clusters of galaxies, WEF obtained the constraint $\sigma_{8} \Omega_{0}^{0.56} \approx 0.57$, for a spatially flat universe. A similar constraint can be obtained from the observed masses and correlation 
functions. As discussed in WEF, the Lagrangian radius of rich clusters, $R$, is about $r_{8} \equiv 8 h^{-1} \mathrm{Mpc}$. For $R$ near $r_{8}$ the rms mass fluctuation, $\sigma(R)$, can be approximated by

$$
\sigma(R) \approx \sigma_{8}\left(r_{8} / R\right)^{\gamma}
$$

where the index $\gamma$ measures the local slope of the fluctuation spectrum, and is given by $\gamma=0.68+0.4 \Gamma$ for the CDM-like spectra discussed here (Efstathiou, Bond \& White, 1992a). Using equation (9) and equation (7) we obtain

$$
\sqrt{\frac{\xi_{\mathrm{cc}}(r)}{\xi_{\mathrm{N}}(r)}}=\sigma_{8}\left(1-\frac{1}{\delta_{c}}\right)+\delta_{c}\left[\frac{M}{M_{8}}\right]^{2 \gamma / 3} \frac{1}{\sigma_{8} \Omega_{0}^{2 \gamma / 3}},
$$

where $\xi_{\mathrm{N}}$ is the mass two-point correlation function for a linear power spectrum with $\sigma_{8}=1 ; M_{8}=5.8 \times 10^{14} h^{-1} M_{\odot}$, as introduced in Section 2. For rich clusters, the first term in the r.h.s. of equation (10) can be neglected, and it follows that

$$
\sigma_{8} \Omega_{0}^{2 \gamma / 3}=\delta_{c}\left[\frac{M}{M_{8}}\right]^{2 \gamma / 3}\left[\frac{\xi_{\mathrm{cc}}}{\xi_{\mathrm{N}}}\right]^{-1 / 2} .
$$

For rich clusters, $M \approx M_{8}(\mathrm{WEF})$ and $\left(\xi_{\mathrm{cc}} / \xi_{\mathrm{N}}\right) \approx 10$. We get $\sigma_{8} \Omega_{0}^{0.5} \approx 0.53$ for $\Gamma=0.2$. This result is very similar to that obtained by WEF from cluster abundances.

Combining this result (or the result of WEF) with our result for $\sigma_{8}$, one can in principle get an estimate for $\Omega_{0}$. Indeed, if the power spectrum of mass density fluctuations has the shape suggested by the APM survey (i.e. $\Gamma \approx 0.2)$, then $r_{0} \gtrsim 20 h^{-1} \mathrm{Mpc}$ for Abell clusters would require $\Omega_{0} \lesssim 0.3$, while $r_{0} \lesssim 15 h^{-1} \mathrm{Mpc}$ would require $\Omega_{0} \gtrsim 1$. With better data on cluster masses and correlations, these arguments should provide an estimate of $\Omega_{0}$. 


\section{DISCUSSION}

Our simulations show that the mass function and the autocorrelation function of clusters can be quite accurately predicted by our Press-Schechter model. As a result this model provides a simple and intuitive way to understand how cluster data constrain $\sigma_{8}$ and $\Omega_{0}$. A significant uncertainty remains, however, in the operational definition of a galaxy cluster. In our model, as in most theoretical studies, clusters are selected according to mass and overdensity thresholds. The two group-finding algorithms we have tested give similar mass functions and two-point correlation functions. Unfortunately, the selection criteria in real observational samples can be far more complex than assumed by these algorithms, and may differ from catalogue to catalogue. In a recent paper, Eke et al. (1995) used N-body simulations of the SCDM cosmogony to examine how the two-point correlation function of a cluster sample depends on the way it is selected. They found small but significant variations between cluster samples defined in different ways. Their results suggest that selection effects must be considered carefully, when a rigorous comparison between models and observations is made. In the future, when cluster samples with well defined selection criteria are available, one should use simulations that take into account these selection criteria in as much detail as possible in order to calibrate the kind of measurements we are suggesting. The observed masses and correlations of rich clusters should then provide direct and independent measurements of the density of the Universe and of the amplitude of mass fluctuations. 


\section{Acknowledgements:}

We thank S. Cole and C. Lacey for providing us with their code for the SO group finder. YPJ acknowledges the receipt of an Alexander-von-Humboldt research fellowship. 


\section{REFERENCES}

??bell G.O., 1958, ApJS, 3, 211

??bell G.O., Corwin H.G., Olowin R.P., 1989, ApJS, 70, 1

??ahcall N.A., 1988, ARA\&A, 26, 631

??ahcall N. A., Burgett W., 1986, ApJL, 300, L35

??ahcall N. A., Cen R.Y., 1992, ApJ, 398, L81

??ahcall N. A., Soneira R. M., 1983, ApJ, 270, 20

??ahcall N. A., West M., 1992, ApJ, 392, 419

??ardeen J., Bond J.R., Kaiser N., Szalay A.S., 1986, ApJ, 304

??ond J.R., Cole S., Efstathiou G., Kaiser N., 1991, ApJ, 379, 440

??ole S., Kaiser N., 1989, MNRAS, 237, 1127

??roft R.A.C., Efstathiou G., 1994, MNRAS, 267, 390

??alton G.B., Efstathiou, G., Maddox S.J., Sutherland W.J., 1992, ApJ, 390, L1

??alton G.B., Croft R.A.C., Efstathiou, G.,Sutherland W.J., Maddox S.J., Davis M., 1994, MNRAS, 271, L47

??avis M., Efstathiou G., Frenk C.S., White S.D.M., 1985, ApJ, 292, 371

??ekel A., Blumenthal G. R., Primack J. R., Olivier S., 1989, ApJ, 338, L5

??fstathiou G., Bond J.R., White S.D.M., 1992a, MNRAS, 258, $1 \mathrm{P}$

??fstathiou G., Dalton G.B., Sutherland W.J., Maddox S.J., 1992b, MNRAS, 257, 125

??fstathiou G., Frenk C.S., White S.D.M., Davis M., 1988, MNRAS, 235, 715

??fstathiou G., Sutherland W.J., Maddox S.J., 1990, Nat, 348, 705

??ke V.R., Cole S., Frenk C.S., Navarro J.F., 1995, MNRAS, in press 
??unn J. E., Gott J. R., 1972, ApJ, 176, 1

??ing Y.P., Mo H.J., Börner G., Fang L.Z., 1993, ApJ, 411, 450

??ing Y.P., Mo H.J., Börner G., Fang L.Z., 1995, MNRAS, 276, 417

??ing Y.P., Plionis M., Valdarnini R., 1992, ApJ, 389, 499

??aiser N., 1984, ApJL, 284, L9

??ashlinsky M., 1987, ApJ, 317, 19

??lypin A., Kopylov A., 1983, Sov. Astron. Lett., 9, 41

??acey C., Cole S., 1994, MNRAS, 271, 676

??ann R.G., Heavens A.F., Peacock J.A., 1993, MNRAS, 263, 798

??o H.J., Jing Y.P., Börner G., 1992, ApJ, 392, 452

??o H.J., Peacock J.A., Xia X.Y., 1993, MNRAS, 260, 121

??o H.J., White S.D.M., 1995, preprint

??ichol R.C., Collins C.A., Guzzo L., Lumsden S.L., 1992, MNRAS, 255, $21 \mathrm{P}$

??eacock J.A., West M.J., 1992, MNRAS, 259, 494

??eebles P.J.E., 1980, The Large-Scale Structure of the Universe, Princeton University Press, Princeton

??ostman M., Huchra J.P., Geller M.J., 1992, ApJ, 384, 404

??ress W.H., Schechter P., 1974, ApJ, 187, 425 (PS)

??moot G.F. et al. 1992, ApJ, 396, L1

??utherland W., 1988, MNRAS, 234, 159

??hite S.D.M., Frenk C.S., Efstathiou G., 1993, MNRAS, 262, 1023 (WEF)

??hite S.D.M., Frenk C.S., Davis M., Efstathiou G., 1987, ApJ, 313, 505

??right E.L., 1994, ApJ, 420, 1 

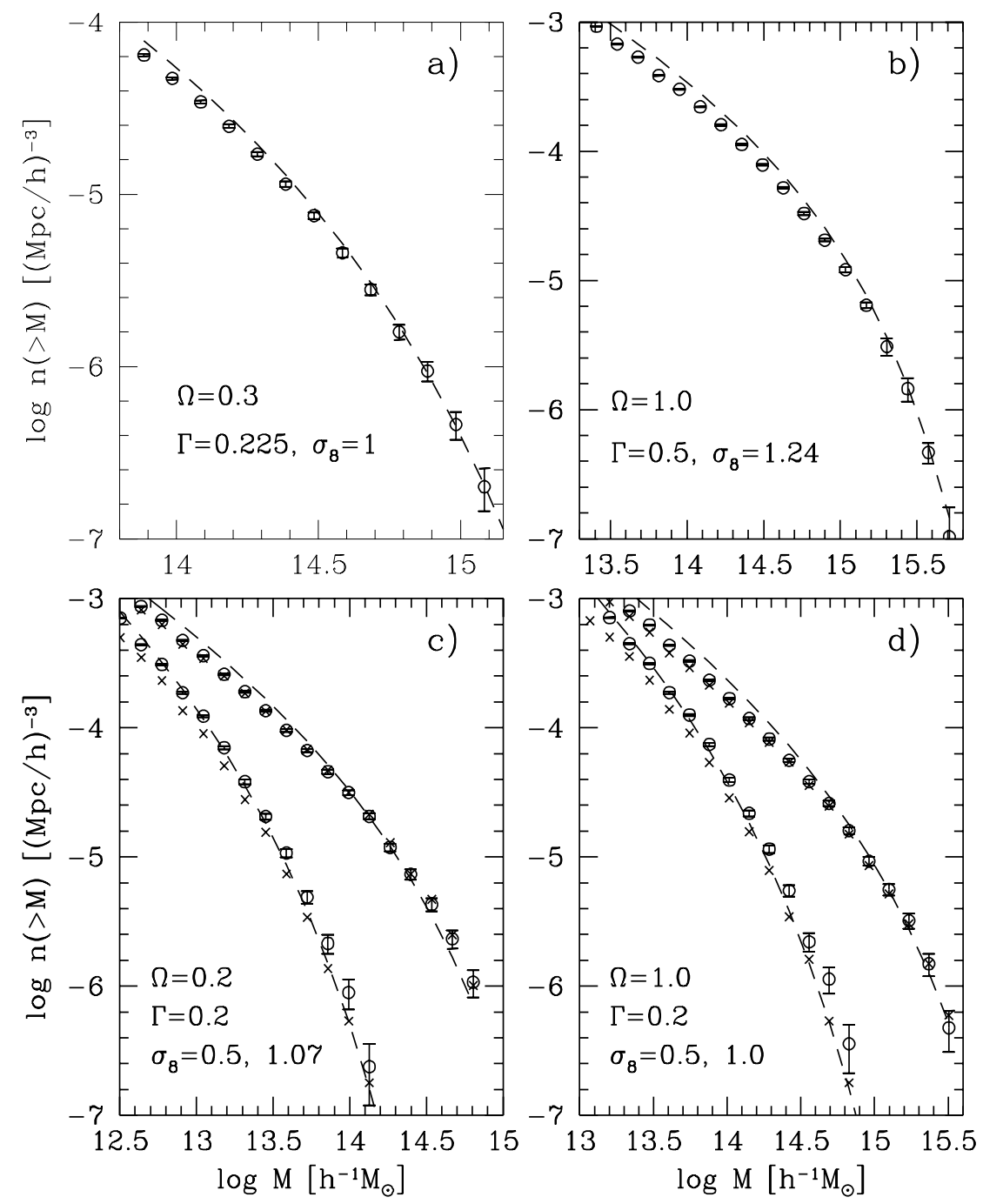

Figure 1. Cumulative mass function of FOF clusters in N-body simulations (circles), compared to the predictions of the PS formalism (dashed curves). Error bars in (a) and (b) denote the scatter between different realizations; those in (c) and (d) denote Poisson errors in the number density. In (c) and (d), higher curves correspond to higher values of $\sigma_{8}$. For comparison, results for $\mathrm{SO}$ clusters are also ploted as crosses in (c) and (d). 


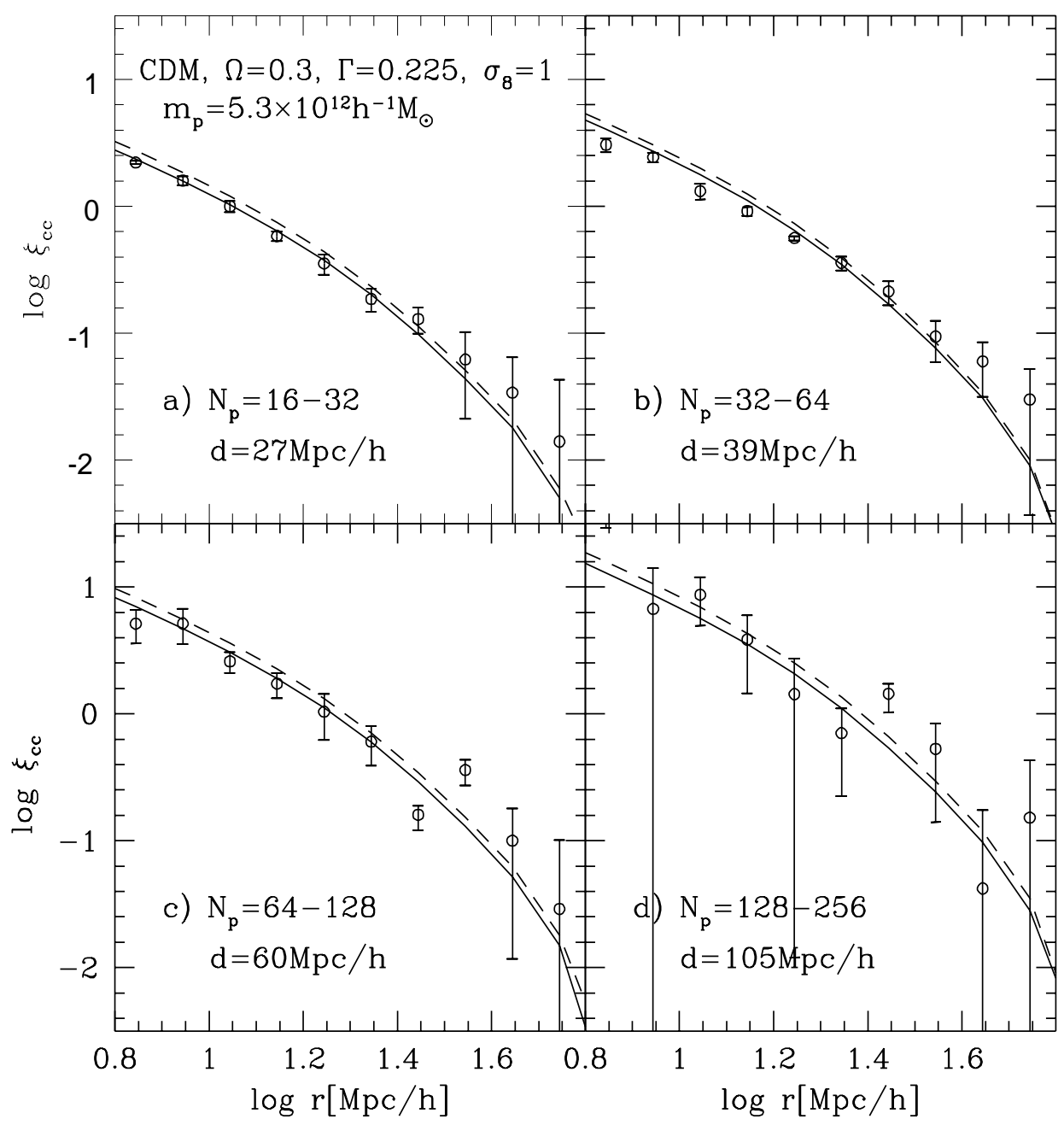

Figure 2. Two-point correlation functions of clusters of galaxies identified by the FOF group finder in N-body simulations (circles), compared to model predictions (dashed curves). This is for a model with $\left(\Omega_{0}, \Gamma, \sigma_{8}\right)=(0.3,0.225,1)$. Solid curves show fits of the data points at $r>10 h^{-1} \mathrm{Mpc}$ to a model in which $\xi_{\mathrm{cc}}(r)$ is proportional to the two-point correlation function corresponding to the linear power spectrum. The error bars represent the $1 \sigma$ scatter between different realizations. The value of $m_{p}$ denotes the mass of a single mass particle in the simulation. Results are shown for clusters in different mass ranges, as indicated by the number of particles, $N_{p}$, contained in these clusters. The value of $d$ denotes the mean intercluster separation for clusters with masses exceeding the lower limit of each mass bin. 


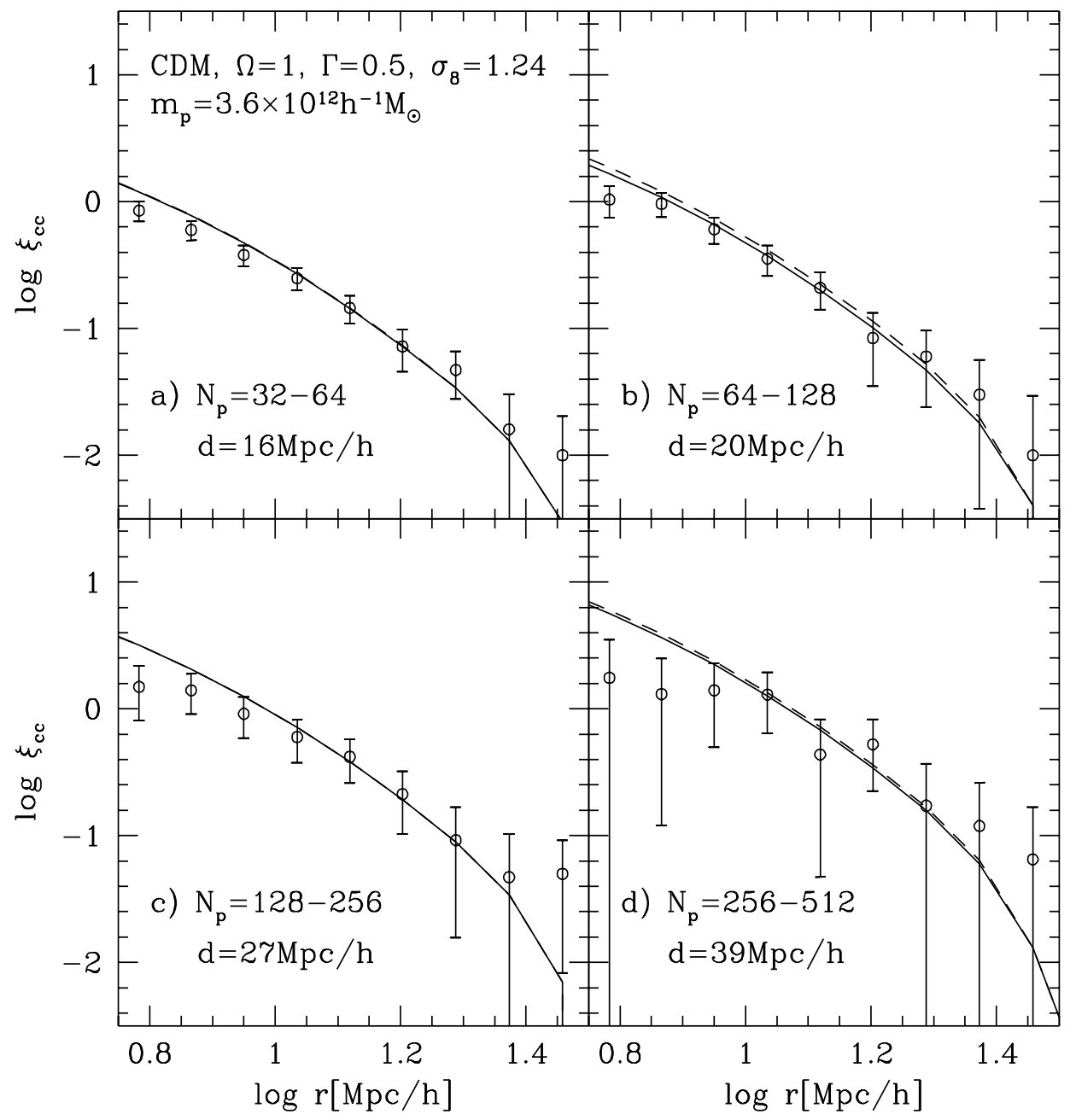

Figure 3. The same as Figure 2, for a model with $\left(\Omega_{0}, \Gamma, \sigma_{8}\right)=(1,0.5,1.24)$. 


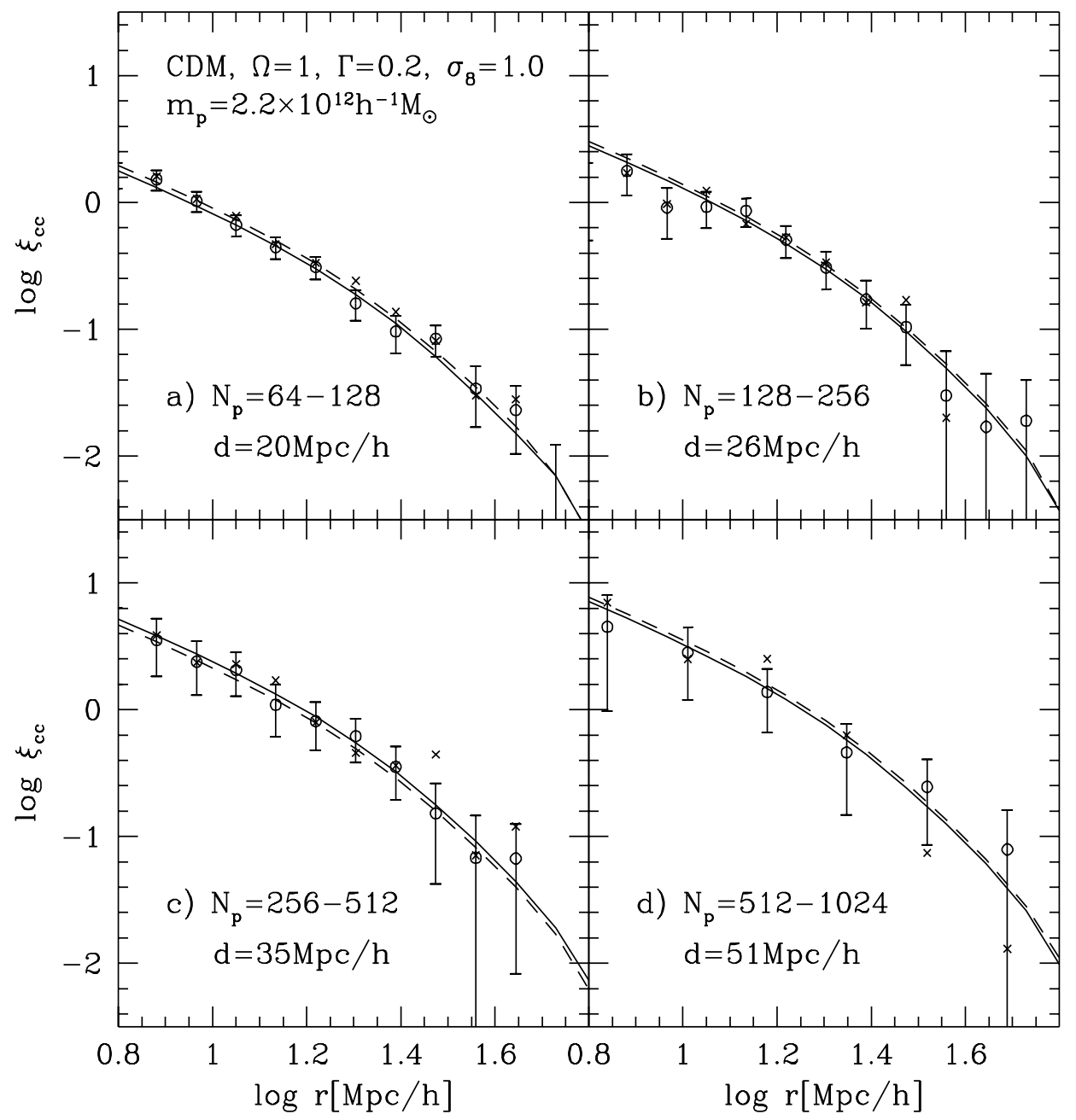

Figure 4. The same as Figure 2, for a model with $\left(\Omega_{0}, \Gamma, \sigma_{8}\right)=(1,0.2,1)$. Unlike in Fig.2, error bars here denote Poisson fluctuations. Crosses show the same results for $\mathrm{SO}$ clusters. 


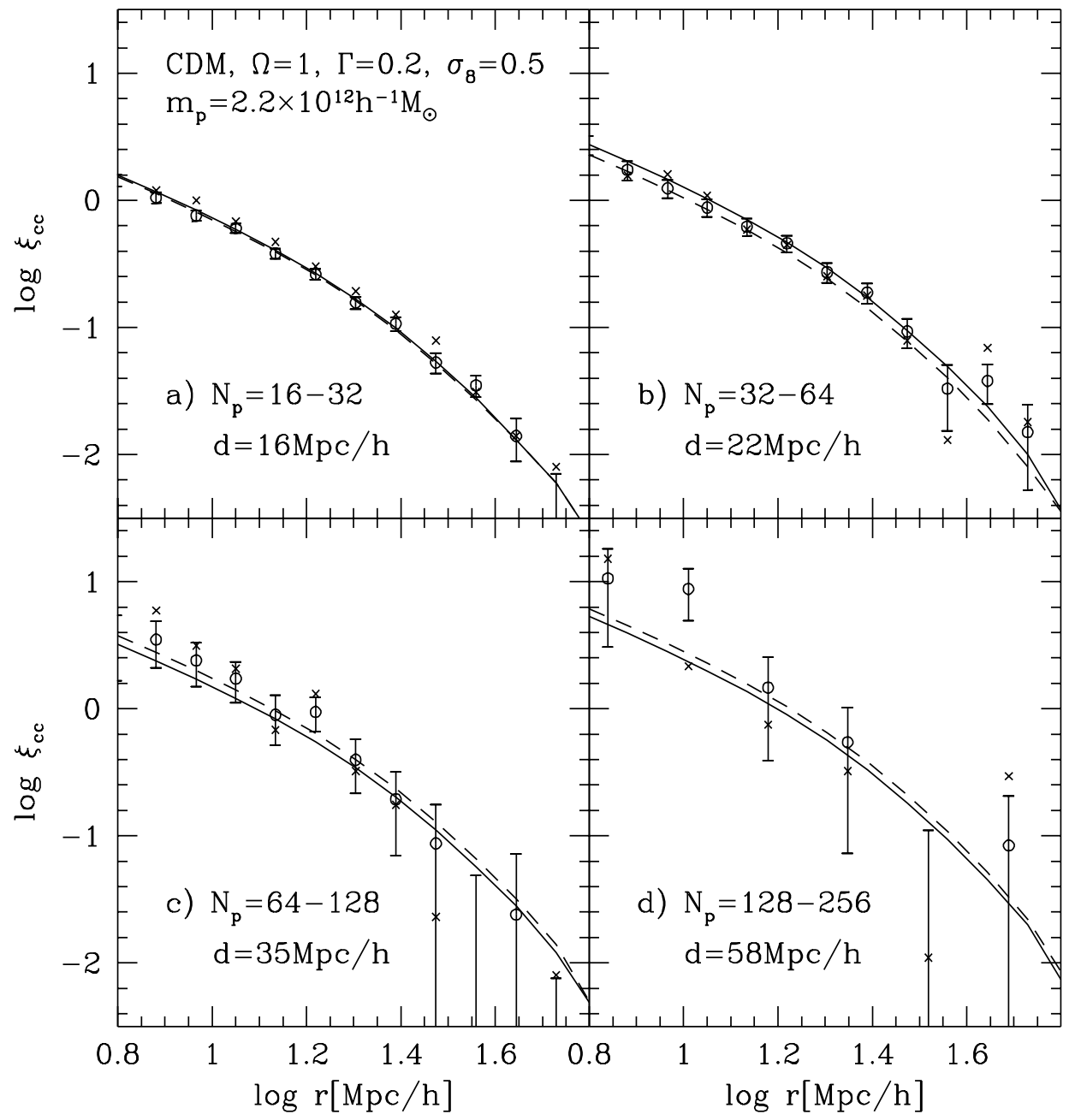

Figure 5. The same as Figure 4, for a model with $\left(\Omega_{0}, \Gamma, \sigma_{8}\right)=(1,0.2,0.5)$. Crosses show the same results for SO clusters. 


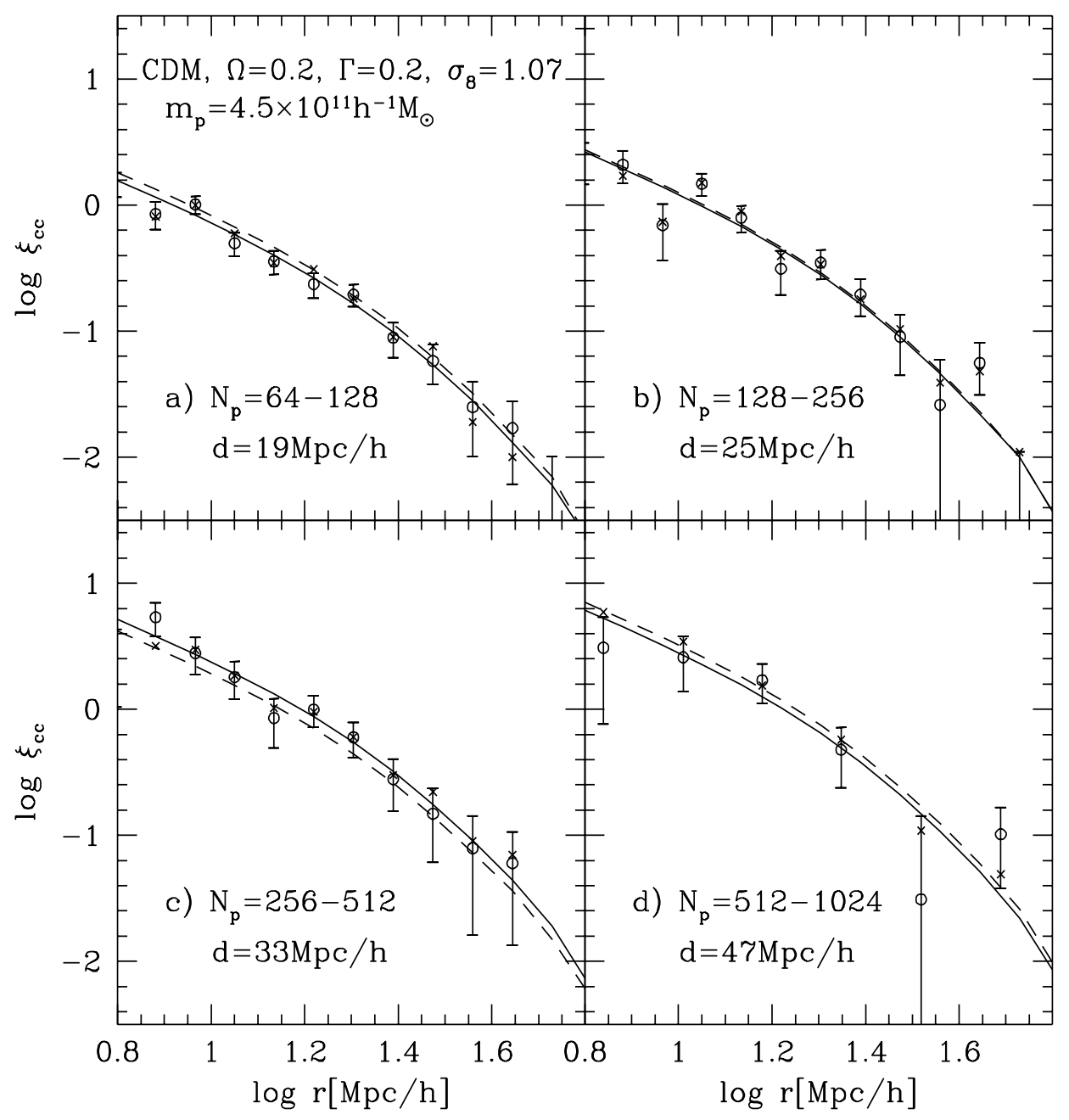

Figure 6. The same as Figure 4, for a model with $\left(\Omega_{0}, \Gamma, \sigma_{8}\right)=$ $(0.2,0.2,1.07)$. Crosses show the same results for SO clusters. 


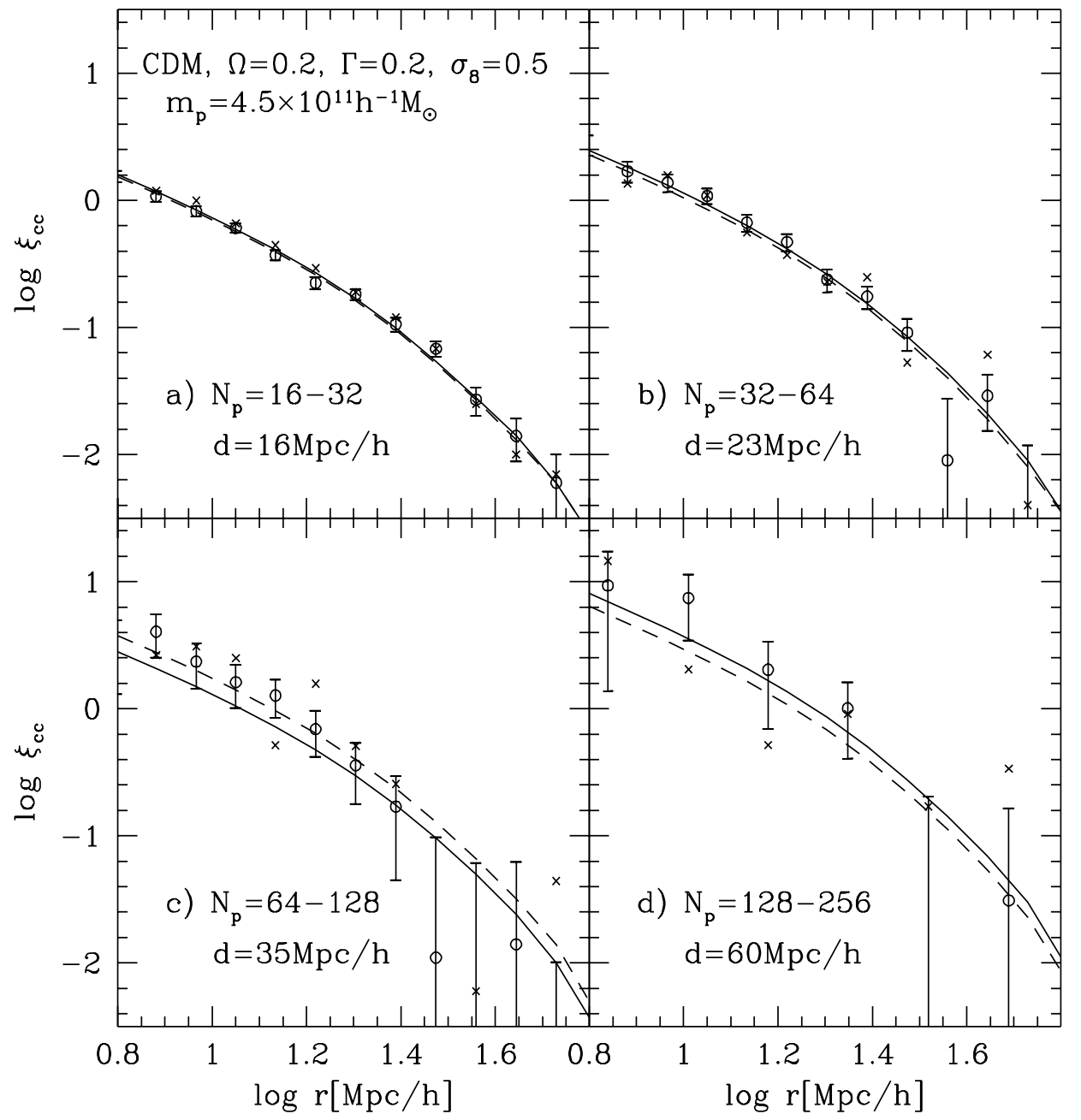

Figure 7. The same as Figure 4, for a model with $\left(\Omega_{0}, \Gamma, \sigma_{8}\right)=(0.2,0.2,0.5)$. Crosses show the same results for SO clusters. 


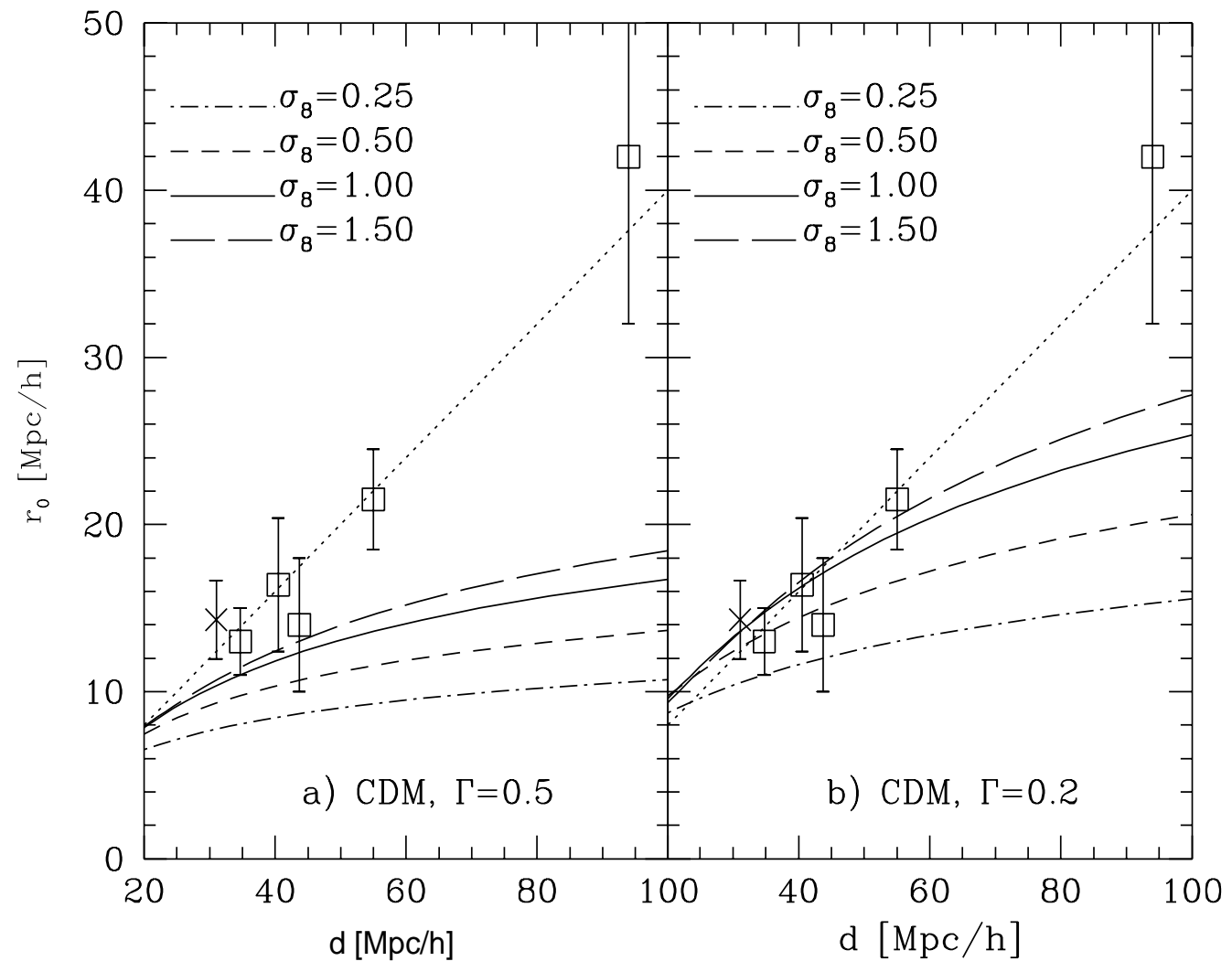

Figure 8. Correlation length $r_{0}$ as a function of the mean intercluster separation $d$, for two sets of models with $\Gamma=0.5$ (a) and $\Gamma=0.2$ (b). For a given $\Gamma$, the $d-r_{0}$ relation does not depend on $\Omega_{0}$, but only on $\sigma_{8}$. The data points (squares) are observational results compiled by Bahcall \& Cen (1992), and the error bars are $1 \sigma$. In addition, the result of a recent determination by Dalton et al. (1994) for APM clusters is also plotted (the cross) together with its $2 \sigma$ error bar. The dotted lines show the relation $r_{0}=0.4 d$. 